\title{
A NOTE ON SPLIT DILATIONS DEFINED BY HIGHER RESIDUES
}

JAY P. FILLMORE

1. Introduction. Let $p$ be an odd prime, $F_{p}$ the field of $p$ elements. Given $a, b \in F_{p}$ the mapping

$$
\begin{aligned}
x \rightarrow 0 & \text { if } x=0, \\
\rightarrow a x & \text { if }(x / p)=1, \\
\rightarrow b x & \text { if }(x / p)=-1 .
\end{aligned}
$$

where $(x / p)$ is the quadratic residue symbol, has been called a split dilation by W. H. Mills. If $a b \neq 0$ and $(a / p)=(b / p)$, it is a permutation of the elements of $F_{p}$.

Two maps $P, Q$ of $F_{p}$ into itself are called equivalent if $Q(x)$ $=P(x+a)+b$ for some $a, b$ and all $x$ in $F_{p}$. Two split dilations have the property that they are equivalent only when they are equal.

In this note we discuss split dilations of a field with $q$ elements where the splitting is defined for any divisor of $q-1$. Such split dilations are equivalent only when they are equal. The attractive conjecture that every permutation is equivalent to some split dilation is shown false by enumerating the split dilations and comparing this with the total number of equivalence classes.

2. Split dilations. Let $F$ be the field with $q$ elements and characteristic $p, d$ a divisor of $q-1, \kappa$ a primitive $d$ th root of unity in $F$, $r=(q-1) / d$.

Let $a=\left(a_{1}, \cdots, a_{d}\right) \in F^{d}$. The map $D_{a}$ defined by

$$
\begin{aligned}
D_{a}(x) & =0 & & \text { if } x=0 \\
& =a_{1} x & & \text { if } x^{r}=\kappa \\
& =a_{2} x & & \text { if } x^{r}=\kappa^{2} \\
& \vdots & & \vdots \\
& =a_{d} x & & \text { if } x^{r}=\kappa^{d}=1
\end{aligned}
$$

is said to be a $d$-split dilation of $F$.

Every mapping of $F$ into itself is given by a unique polynomial of degree less than $q$. To see that a $d$-split dilation $D_{a}$ may be put into the form

Received by the editors May 2, 1966. 


$$
D_{a}(x)=A_{d-1} x^{r(d-1)} x+A_{d-2} x^{r(d-2)} x+\cdots+A_{1} x^{r} x+A_{0} x
$$

it is only necessary to solve the system

$$
\sum_{i=0}^{d-1} A_{i} \kappa^{j i}=a_{j}, \quad j=1,2, \cdots, d .
$$

The determinant is a nonvanishing Vandermonde determinant and the system has a solution.

Call two maps $P, Q$ of $F$ into itself equivalent if there are $\alpha, \beta \in F$ such that $Q(x)=P(x+\alpha)+\beta$ for all $x$. If $Q$ and $P$ are written as polynomials of degree less than $q$, their degrees are equal (the zero polynomial being assigned the degree 0 ).

TheOREM 1. Let $d \mid q-1, d \neq q-1$. Let $D_{a}$ be $d$-split and suppose the degree of the polynomial representing $D_{a}$ is not divisible by $p$. Then any $d$-split dilation which is equivalent to $D_{a}$ equals $D_{a}$.

Proof. Suppose $D_{a}(x)$ is given by the polynomial

$$
A_{s-1} x^{r(s-1)+1}+A_{s-2} x^{r(s-2)+1}+\cdots+A_{1} x^{r+1}+A_{0} x
$$

where $A_{s-1} \neq 0$ and $1 \leqq s \leqq d$. Then $D_{a}(x+\alpha)+\beta$ is given by the polynomial

$$
A_{s-1} x^{r(s-1)+1}+(r(s-1)+1) A_{s-1} \alpha x^{r(s-1)}+\cdots .
$$

If $s=1$ the theorem is trivial, so assume that $s>1$.

Since $d \neq g-1, r(s-1)>r(s-2)+1$ and the coefficient of the $r(s-1)$ st degree term is $(r(s-1)+1) A_{s-1} \alpha$. If $D_{a}(x+\alpha)+\beta$ is to be $d$-split, this coefficient $=0$. Since $r(s-1)+1 \neq 0, A_{s-1} \neq 0$, it follows $\alpha=0$. $\beta=0$ comes from setting $x=0$.

REMARK. The hypothesis on the degree of the polynomial representing $D_{a}$ is satisfied in two important instances:

$1^{\circ} q=p$,

$2^{\circ}$ the degree of the polynomial representing $D_{a}$ is maximal, i.e., the degree is $r(d-1)+1=(q(d-1)+1) / d$ and hence never $\equiv 0 \bmod p$.

Special cases of split dilations are:

(1) $p$ arbitrary, $d=1 . D_{a}(x)=a_{1} x$ is a dilation of $F$ in the usual sense.

(2) $p$ odd, $d=2, D_{a}(x)=0$ if $x=0, a_{1} x$ is $x^{(q-1, / 2}=-1$, and $a_{2} x^{\prime \prime}$ if $x^{(q-1) / 2}=1\left(a_{1} a_{2} \neq 0, a_{1}^{(q-1) / 2}=a_{2}^{(q-1) / 2}\right)$ is a split dilation as defined by Mills.

(3) $p$ arbitrary, $d=q-1 . D_{a}$, for suitable choice of the $a_{i}$, can be any map of $F$ in to itself which sends 0 in to 0 .

3. The group of split dilations. Let $K_{i}=\left\{x \in F-\{0\} \mid x^{r}=\kappa^{i}\right\}$ 
$i=1, \cdots, d$ (notation as in $\S 2) . K_{d}$ is a subgroup of $F-\{0\}$, the $K_{i}$ are its cosets.

Let $D_{a}, a=\left(a_{1}, \cdots, a_{d}\right)$, be $d$-split. Suppose $a_{i} \in K_{\pi(i)-i}$ where the map $\pi$ of $1,2, \cdots, d$ into itself is determined by $a$. If $x \in K_{i}$, then $D_{a}(x)=a_{i} x \in K_{\pi(i)}$. Furthermore, the map $x \rightarrow a_{i} x$ from the set $K_{i}$ to the set $K_{\pi(i)}$ is bijective. Thus $D_{a}$ is a permutation if, and only if, $\pi$ is a permutation of $1,2, \cdots, d$. Clearly every $\pi$ in the symmetric group $S_{d}$ comes from some $D_{a}$.

Let $D_{a}, D_{a^{\prime}}$ be $d$-split dilations which permute the classes $K_{i}$ according to $\pi, \pi^{\prime}$ respectively. Then $D_{a^{\prime}} \cdot D_{a}$ is a $d$-split dilation $D_{a^{\prime \prime}}$ where $a_{i}^{\prime \prime}=a_{\pi(i)} \cdot a_{i}, i=1,2, \cdots, d . D_{a^{\prime \prime}}$ permutes the $K_{i}$ according to $\pi^{\prime} \cdot \pi$. If we denote by $G_{d}$ the $d$-split dilations of $F$ which are permutations we have:

THEOREM 2. $G_{d}$ is a group of order $d !((q-1) / d)^{d}$. It is a subgroup of index $d^{d}$ of the semidirect product by $S_{d}$ of $d$ copies of the multiplicative group of $F$.

The order of $G_{d}$ is computed by knowing that $S_{d}$ contains $d$ ! elements and that each class $K_{i}$ contains $(q-1) / d$ elements. The other assertions follow easily from the preceding discussion.

Now let $\omega$ be a primitive $(q-1)$ st root of unity in $F$. Put $\kappa_{d}=\omega^{(q-1) / d}$ so that $\kappa_{d}$ is a primitive $d$ th root of unity.

Let $d\left|d^{\prime}, d^{\prime}\right| q-1$. There is then a natural inclusion $G_{d} \subseteq G_{d^{\prime}}$ given by

$$
\begin{aligned}
& D_{\left(a_{1}, \ldots, a_{d}\right)} \rightarrow D_{\left(a_{1}, \ldots, a_{d}, a_{1}, \ldots, a_{d}, a_{1}, \ldots, a_{d}\right)} . \\
& \text { repeated } d^{\prime} / d \text { times }
\end{aligned}
$$

Since $\kappa_{d}=\left(\kappa_{d^{\prime}}\right)^{d^{\prime} / d}$, the two maps agree on all of $F$.

Let $d^{\prime}, d^{\prime \prime}$ be two divisors of $q-1$. Then $G_{d} \subseteq G_{d^{\prime}} \cap G_{d^{\prime \prime}}$ where $d=\left(d^{\prime}, d^{\prime \prime}\right)$ is the greatest common divisor of $d^{\prime}$ and $d^{\prime \prime}$. Our claim is that the inclusion is an equality. This is seen as follows.

Let $P \in G_{d^{\prime}} \cap G_{d}{ }^{\prime \prime}$. Since $P$ is $d^{\prime}$-split, it is given by a polynomial with exponents of the form $k^{\prime}\left((q-1) / d^{\prime}\right)+1\left(k^{\prime}=0,1, \cdots, d^{\prime}-1\right)$ only. Since $P$ is $d^{\prime \prime}$-split, the same holds with exponents $k^{\prime \prime}\left((q-1) / d^{\prime \prime}\right)+1$. Thus each $k^{\prime}\left((q-1) / d^{\prime}\right)+1$ is of the form $k^{\prime \prime}\left((q-1) / d^{\prime \prime}\right)+1$ for suitable $k^{\prime \prime}$. Putting $k=k^{\prime} d / d^{\prime}=k^{\prime \prime} d / d^{\prime \prime}$ we have $k^{\prime}\left((q-1) / d^{\prime}\right)+1$ $=k^{\prime \prime}((q-1) / d)+1=k((q-1) / d)+1$, whence $P$ is also $d$-split. Hence the assertion.

4. Enumerations. By Theorem 2 the number of elements in $G_{d}$ is $d !((q-1) / d)^{d}$. Denote this by $g_{d}$. Let $d_{1}, d_{2}, \cdots, d_{r}$ be the divisors of $q-1$ which are $\neq q-1$. By repeated application of the fact that 
$G_{d^{\prime}} \cup G_{d^{\prime \prime}}$ contains $g_{d^{\prime}}+g_{d^{\prime \prime}}-g_{\left(d^{\prime}, d^{\prime \prime}\right)}$ elements, we obtain

$$
\sum_{\rho=1}^{r}(-1)^{\rho-1} \sum_{1 \leq i_{1}<\cdots<i_{\rho} \leq r} g_{\left(d_{i_{1}}, \cdots, d_{i_{\rho}}\right)}
$$

for the number of elements of

$$
\bigcup_{\rho=1}^{r} G_{d_{\rho}}
$$

If $p=q$, these elements are inequivalent under the equivalence relations defined in $\$ 2$.

Assume now, $p=q$. The $(p-1)$-split dilations are just arbitrary permutations of $F$ which send 0 in to itself. Every equivalence class of permutations of $F$ contains a $(p-1)$-split dilation.

If $p=q=7$, there are 60 permutations which are either $1-, 2-$, or 3 -split, thus 60 equivalence classes containing a permutation which is $d$-split with $d \neq 6$. However, we can compute directly that there are 108 equivalence classes of permutations on the 7 elements of $F_{7}$. Thus equivalence classes containing nontrivial $(d \neq p-1)$ split dilations do not exhaust all equivalence classes.

In fact, for large $p$ equivalence classes containing nontrivial split dilations are quite scarce and their relative number appears to go to 0 as $p \rightarrow \infty$.

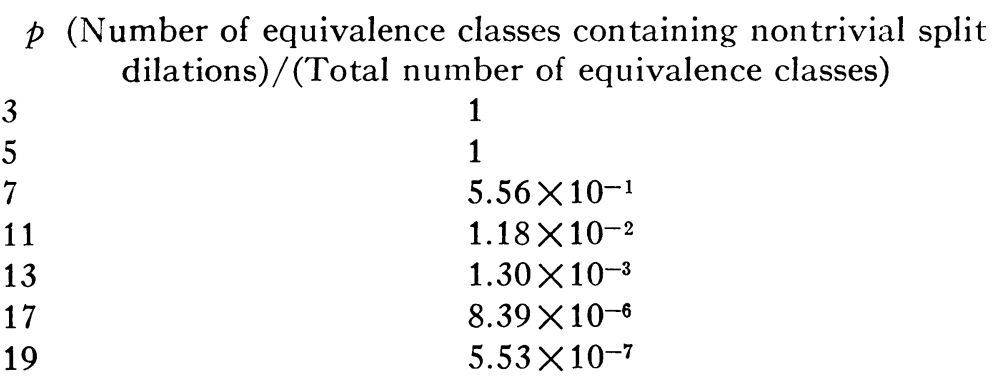

Department of Defense 